\title{
Arbeitskreis Geschichte der Polarforschung
}

\author{
Cornelia Lüdecke ${ }^{1, \text {, }}$ \\ ${ }^{1}$ Universität Hamburg, Hamburg, Deutschland \\ ఓpensioniert
}

Correspondence: Cornelia Lüdecke (c.luedecke@1rz.uni-muenchen.de)

Published: 20 May 2021

\section{Rückblick des AK Geschichte der Polarforschung}

Der Arbeitskreis Geschichte der Polarforschung wurde 1991 auf der 16. Internationalen Polartagung in Göttingen gegründet und wird seitdem von Cornelia Lüdecke geleitet. Unter den Mitgliedern befinden sich neben Polarforschern auch Historiker, Wissenschaftshistoriker und andere Interessenten aus dem In- und Ausland, die Freude an der Geschichte der Polarforschung haben und unterschiedliche Fragestellungen mit Methoden der historischen Forschung an Hand von $\mathrm{Pu}-$ blikationen und unveröffentlichten Quellen bearbeiten.

Grundlegende Aufgaben betreffen das Auffinden bzw. Erschließen von Polarforschernachlässen und das Aufspüren von unveröffentlichten Unterlagen in privaten und öffentlichen Archiven. Gerade Briefwechsel, Tagebücher oder Fotos liefern äußerst wichtige und interessante Grundlagen für die weitere Forschung. Für die jüngere Zeit sind auch verschiedene Ton- und Filmdokumente vorhanden, die es zu sichern und zu sichten gilt. Die Anlage von Bibliographien einzelner Expeditionen oder das Verfassen von Biographien von Expeditionsteilnehmern sind zudem sehr nützliche Hilfsmittel für die Forschung. Die Bewahrung von Originalmessaufzeichnungen und Beobachtungstagebüchern für Vergleiche mit aktuellen Ergebnissen gerade hinsichtlich Klimaveränderung kann sich als lohnenswert erweisen.

Der AK Polargeschichte untersucht nicht nur Fortschritte der einzelnen in der Polarforschung vertretenen Disziplinen oder technische Entwicklungen in der Lösung logistischer Herausforderungen, sondern auch die unterschiedlichen nationalen und internationalen Forschungskooperationen und Schritte zur Institutionalisierung der Polarforschung. Sozialwissenschaftliche und psychologische Betrachtungen können Aufschluss geben darüber, wie die Interaktion innerhalb von Expeditionen und Projekten ablief, um daraus für die Zukunft nützliche Schlussfolgerungen zu ziehen. Interessant ist auch zu sehen, wie historische Ereignisse geisteswissenschaftlich umgesetzt werden wie beispielsweise die in München 2016 uraufgeführte sogenannte Doppeloper in zwei Teilen „South Pole“ komponiert von Miroslav Srnka, während das Libretto von Tom Holloway stammt. Ein anderes Beispiel liefert die literarische Darstellung von Alfred Wegeners Biographie in dem Sachroman von Jo Ländle „Alles Land“ (München, 2011).

Aufgrund des multi- und interdisziplinären Ansatzes kann die Geschichte der Polarforschung nicht isoliert betrachtet werden, sondern im politischen, wirtschaftlichen und kulturellen Kontext. Welche Strömungen begünstigten oder vereitelten welche Forschungsprogramme? Welche persönlichen Verbindungen bestanden und welche Gremien haben den Ausschlag zu Expeditionen in die Arktis oder Antarktis gegeben? Wer hat wen gefördert oder behindert? Die einzelnen Forschungsergebnisse des AK werden vorzugsweise in der Zeitschrift „Polarforschung“ veröffentlicht. Daneben sind Mitglieder des AK Ansprechpartner für Rundfunkinterviews und Fernsehproduktionen oder auch selbst Organisatoren für Ausstellungen.

Um Anregungen für künftige Forschungsarbeiten zu geben, widmeten sich jährliche Rundbriefe jeweils einem Schwerpunkt und ergänzten neues Material für die bestehenden Themen. Seit 1996 erschien der Rundbrief zweisprachig (deutsch/englisch).

1/92 Bibliographie

2/93 Archive

3/94 Hilfsmittel

4/95 Internationale Kooperationen

5/96 Polarphilatelie/Polarphilately

6/97 Polarfilme/Polar Movies 
7/98 Polarbelletristik/Polar Belletristic

8/99 Frauen in Polargebieten/Women in Polar Regions

9/00 Politik und Militär in Polargebieten/Politics and Military in Polar Regions

10/01 10 Jahre Arbeitskreis Geschichte der Polarforschung/10 Years of History of Polar Research Specialist Group

11/02 Logistik: Land-, Luft- und Seefahrzeuge/Logistics: Vehicles, aircraft, and ship

12/03 Deutsche und baltendeutsche Polarforscher in russischen Diensten/German and German-Baltic polar researchers in Russian service

13/04 Kulturelles Erbe - Denkmalschutz in Polargebieten/Culturel heritage - preservation in polar regions

14/05 Biographien/Biographies

15/06 Institutionalisierung der Polarforschung/Institutionalisation of Polar Research,

16/07 Die Internationalen Polarjahre 1882-1883, 19321933 und 1957-1958/International Polar Years 18821883, 1932-1933, and 1957-1958

\section{7/08 Polare Kunst/Polar Art}

Die Rundbriefe, die künftig auf der Homepage der Gesellschaft für Polarforschung unter AK Geschichte der Polarforschung abrufbar sind, sollten insbesondere Anregung geben, sich näher mit einzelnen Themen zu beschäftigen. Zusätzlich wurden neben Tagungsberichten und einer Bibliographie von Artikeln mit Bezug zur deutschen Polargeschichte auch aktuelle Informationen gegeben über Archive, Ausstellungen oder kommende Tagungen. Darüber hinaus wurde aufgelistet, welche Vorträge oder Poster von AK-Mitgliedern im vergangenen Jahr gehalten bzw. auf Tagungen präsentiert wurden. Einen großen Raum nahmen zum Abschluss der Rundbriefe die anstehenden Jubiläen des kommenden Jahres ein, die im Abstand von 25 Jahren jeweils Geburtstage, Todestage und Expeditionsereignisse des nächsten Jahres einschlossen. Mit der Einführung des Internets wurden die Rundbriefe jedoch überholt.

Davon abgesehen organisiert der AK während der Polartagungen Vorträge zu historischen Themen und fördert den wissenschaftlichen Austausch in seiner Mitgliederversammlung. Gelegentlich beteiligt er sich an anderen größeren Veranstaltungen oder organisiert eigene Tagungen in Zusammenarbeit mit dem Fachausschuss Geschichte der Meteorologie der Deutschen Meteorologischen Gesellschaft.

Zunächst fand von 24.-25. September 2011 anlässlich des hundertsten Jubiläums der Expedition des Herzogs Ernst II von Sachsen-Altenburg nach Spitzbergen die Tagung „Von
A(ltenburg) bis Z(eppelin): Deutsche Forschungen in Spitzbergen bis 1914“ mit zehn Vorträgen statt. Während der öffentlichen Abendveranstaltung wurde nach einer Einführung die Fernsehdokumentation „Verschollen vor Spitzbergen“, ein Film über die gescheiterte Vorexpedition von Herbert Schröder-Stranz nach Spitzbergen 1912-1913 gezeigt. Die Vorträge wurden in der Schriftenreihe des Instituts für Geodäsie der Universität der Bundeswehr München, Neubiberg, Heft 88, (2012) veröffentlicht.

Vom 6.-7. Oktober 2016 organisierten beide Gruppen im Zeppelin Museum in Friedrichshafen eine Tagung anlässlich des 85. Jahrestages der Arktisfahrt des LZ 127 „Graf Zeppelin“ unter dem Thema „Die Erforschung der Arktis aus der Luft" mit zehn Vorträgen. Dazu wurde ein öffentlicher Abendvortrag gehalten über „Norge“, „Italia“ und „Graf Zeppelin“ 1926-1931: Fünf Jahre Luftschiffe in der Arktis“, dem der 15 minütige Schwarzweißfilm mit dem Titel „Mit Graf Zeppelin in die Arktis (1931)“ folgte. Den Abschluss der Tagung bildete eine Führung durch das Zeppelin Museum. Vier Beiträge der Zeppelintagung wurden in der Polarforschung $88 \mathrm{Nr}$. 1, (2018) publiziert.

Immer wieder werden Anfragen für Radiointerviews und zu Fernseh- oder Buchdokumentationen an den AK gerichtet. Dank diverser Jubiläen wird die Bedeutung der Polargeschichte zunehmend wahrgenommen. Beispielsweise wurde von Springer ein Buch über die Geschichte der Polarjahre herausgegeben (Barr und Lüdecke, 2010) an dem sich auch AK-Mitglieder beteiligten. Es entstehen geisteswissenschaftliche Dissertationen über die deutsche Polarforschung (z. B. Schilling, 2016). Der AK Polargeschichte gibt bei solchen Projekten gerne Auskünfte oder Hinweise. Ebenso treten AK-Mitglieder in verschiedenen Formaten in der Öffentlichkeit als Dozenten mit Themen zur Polargeschichte auf. An den Arbeiten des AK kann sich jeder beteiligen, der sich für die Geschichte der Polarforschung interessiert.

\section{Interview mit Prof. Dr. Cornelia Lüdecke}

Bereits 1991 hat Prof. Dr. Cornelia Lüdecke den Arbeitskreis Geschichte der Polarforschung ins Leben gerufen. Im Interview berichtet sie über die verschiedenen Quellen, die sie als Polarhistorikerin untersucht, und warum eine Meuterei auf der ,Deutschland“ gerade noch glimpflich ausging.

APECS Germany: Kurz zu Ihrer Person und Ihrem beruflichen Werdegang. Wollten Sie schon immer Polarforscherin werden? Wann und warum haben Sie sich für die Polarforschung entschieden?

C. Lüdecke: Nach meinem Diplomabschluss in Meteorologie bin ich durch Literaturrecherchen über die physikalischen Eigenschaften von Meereis für die MAN Neue Technologie zur Polargeschichte gelangt. Dies führte mich zu einem Zweitstudium Geschichte der Naturwissenschaften und meiner Dissertation über die Geschichte der deutschen Polarforschung im Zeitraum 1900 bis zum Zweiten Weltkrieg 
und den Einfluss Erich von Drygalskis, dem Leiter der ersten deutschen Südpolarexpedition (1901-1903). Nachdem die Polargeschichte so spannend ist, bin ich dabei geblieben.

APECS Germany: Warum setzen Sie sich für das Thema Ihres AK ein? Was macht dieses Forschungsfeld für Sie so besonders, dass Sie dafür so viel Zeit und Einsatz bringen?

C. Lüdecke: Ich habe den AK Geschichte der Polarforschung 1991 in der Gesellschaft eingerichtet, um das Interesse für die Geschichte und die professionelle Behandlung anzuregen und zu fördern.

APECS Germany: Was gefällt Ihnen an Ihren Aufgaben als AK Leiter ganz besonders? Hat sich dadurch Ihre Sicht auf etwas geändert?

C. Lüdecke: Als Leiterin des AK kann ich Leute anregen, auf Tagungen Vorträge oder Poster beizusteuern oder Buchrezensionen zu schreiben. Leider gibt es weiterhin von den physikalisch, geologisch oder biologisch arbeitenden Polarforschern viel zu wenig wissenschaftliche Anerkennung für Polarhistoriker, die meist zu den geisteswissenschaftlich arbeitenden Historikern gezählt werden. Was dem Polarforscher im üblichen Sinn seine Messdaten bedeuten, sind dem Polarhistoriker die Quellen, d.h. meist Schriftstücke.

APECS Germany: Wenn Sie in die Zukunft schauen, was würden Sie gerne im Feld Ihres Arbeitskreises in fünf Jahren herausgefunden haben? Was ist in Ihren Augen die dringendste Frage im Feld Ihres Arbeitskreises?

C. Lüdecke: Beispielsweise könnte man untersuchen, ob und wie sich das Umweltverständnis deutscher Expeditionen in Polarregionen seit den Anregungen von August Petermann für Arktisexpeditionen und Georg von Neumayer für Antarktisexpeditionen verändert hat. Dazu gehört auch das Herausarbeiten von Hinweisen auf mögliche Klimaänderungen in historischen und nicht geologischen Zeiträumen.

APECS Germany: Was darf für Sie bei der Archivarbeit nie fehlen?

C. Lüdecke: Sehr nützlich sind gute Hinweise auf Quellen, wo man noch nachschauen könnte, um gewisse Fragestellungen beantworten zu können. Vor allem sind Hinweise auf Nachlässe von Polarforschern im Privatbesitz hochinteressant, denn da kann man auf Briefwechsel, Tagebücher, Berichte und Fotos stoßen.

APECS Germany: Was war Ihre einprägsamste (lustig/beängstigend/seltsam) Erfahrung in der Polarforschung?

C. Lüdecke: Die tollste Erfahrung in der Recherche für meine Dissertation über die deutsche Polargeschichte hatte ich, als ich im Archiv von Auswärtigen Amt in den Akten zu Wilhelm Filchners Antarktisexpedition (1911-1912) auf einen vertraulichen Bericht von Carl Anton Larsen, dem Leiter der Walfangstation Grytviken auf Südgeorgien, gestoßen bin, der über den norwegischen König an Kaiser Wilhelm II gelangte und von dort an das Auswärtige Amt in Berlin und schließlich nach dem Zweiten Weltkrieg zu den Akten des Auswärtigen Amtes in Bonn. Es war, als ob ich unverhofft auf eine Schatzkiste gestoßen war. In diesem Bericht stand, dass es beim Einlaufen der „Deutschland“ in den Hafen von
Grytviken eine Meuterei an Bord gegeben hat, in der sich einige Wissenschaftler, Offiziere und Mannschaftmitglieder lautstark gegen Filchner stellten unter der falschen Annahme, dass sie zusätzlich zu ihrer Heuer keine Polarzulage bekommen würden, weil die Expedition ja nicht in der Antarktis überwintert hatte, sondern an Bord der „Deutschland“ durch das Weddellmeer gedriftet war. Larsen half Filchner, die Meuterer zu beschwichtigen, indem er vorschlug, die Expedition offiziell in Südgeorgien zu beenden. Dadurch würden alle ihre entsprechende Heuer und Zulagen bekommen. Ein Nebeneffekt war die damit unausgesprochene Verpflichtung der Meuterer, nichts über die Vorfälle bekannt zu machen, denn sonst würden sie vor Gericht als Meuterer verurteilt werden nie mehr einen Job bekommen. Das war es also, warum die Expedition nicht wie üblich im Heimathafen beendet wurde und die Gründe dafür im Geheimen blieben.

APECS Germany: Und natürlich eine wichtige Frage zum Schluss: Wer ist Ihr Lieblingspolarforscher oder Ihre Lieblingspolarforscherin?

Mein Lieblingspolarforscher ist Erich von Drygalski, der Leiter der ersten deutschen Südpolarexpedition (19011903), der bei der Vorbereitung auf seine Erfahrungen bei der Überwinterung in Grönland (1892-1893) zurückgreifen konnte und anschließend umsichtig und mit großem Geschick die Expedition in die Antarktis geleitet hat. Meine Lieblingspolarforscherin ist die Amerikanerin Louise Arner Boyd, die hauptsächlich in den 1930er Jahren ihr gesamtes Erbe in fünf von ihr geleitete Expeditionen nach Grönland gesteckt hat, wo sie mit ihren photogrammetrischen Geländeaufnahmen wichtige Grundlagen für amerikanischen Karten der Ostküste Grönlands geliefert hatte.

Das Gespräch führte Johanna Grabow.

Datenverfügbarkeit. Die angesprochenen Rundbriefe sind auf der Homepage der Polargesellschaft verfügbar.

Interessenkonflikt. Die Autor*innen erklären, dass kein Interessenkonflikt besteht.

\section{Literatur}

Barr, S. and Lüdecke, C. (Eds.): The History of the International Polar Years (IPYs), Springer, Heidelberg, Dordrecht, London, New York, 319 pp., 2010.

Schilling, P.: Der letzte weiße Flecken, Wallstein, Göttingen, 448 S., 2016. 